\title{
Breast Cancer Anatomic Stage
}

National Cancer Institute

\section{Source}

National Cancer Institute. Breast Cancer Anatomic Stage. NCI Thesaurus. Code C139445.

Breast cancer staging used in global regions where biomarker tests are not routinely available. 